\section{GROWTH OF INFANT FED STARTER FORMULA CONTAINING PREBIOTICS OR SYMBIOTIC}

\author{
G. Puccio' ${ }^{1}$, C. Cajozzo ${ }^{1}$, F. Meli ${ }^{1}$, G. Licata \\ Ricottone $^{1}$, S. Pecquet ${ }^{2}$, P. Steenhout ${ }^{2}$ \\ 'Universita' degli Studi di Palermo, Palermo, Italy, \\ ${ }^{2}$ Nestle Nutrition, Vevey, Switzerland
}

Objective: To assess growth of infants fed starter formula supplemented with either prebiotics or symbiotic. In addition, gut microbiota was studied.

Methods: Healthy, full term newborns ( $n=240)$ were enrolled before the $14^{\text {th }}$ day of their life and randomly assigned between 3 exclusively formula fed groups: starter IF, starter IF + prebiotics (CMOS+GOS), starter IF + prebiotics (CMOS+GOS) and probiotics (BL999 + LPR). Growth was assessed by body weight gain during 4 months. Gut microbiota profile was examined by measuring fecal counts in Bifidobacteria, Lactobacilli, Enterobacteria, Clostridium at 2 months of age. Incidence of morbidity was appraised.

Results: Non inferiority in growth was established between the two experimental groups and the control at the end of the 4 months intervention period. $90 \%$ confidence intervals for the effects of prebiotics versus control and symbiotic versus control were $(-1.24,3.17)$ and $(-2.35,2.02)$ respectively. Higher levels of fecal Bifidobacteria were observed in the experimental groups; this difference being significant between the symbiotic and control groups $(8.8+/-1.7$ versus $9.87+/-1.2$ $p<0.05)$. Furthermore, Clostridium levels were lower in both experimental vs control groups. No statistically significant differences of adverse events were detected between groups, except for a higher incidence of colics in the prebiotic and, to a lesser degree, in the symbiotic group vs control.

Conclusion: Growth of newborns receiving IF containing prebiotics or symbiotic was non inferior to the growth observed in controls and within the limits defined by WHO growth reference curves. A bifidogenic effect due to symbiotic formulation was demonstrated.

\section{PARENTERAL AMINOACIDS ADMINISTRATION IN PRETERM NEWBORNS OF BIRTH WEIGHT < 1250 G: POSSIBLE ADVANTAGES OF HIGHER INTAKES}

P. Gaio, S. Scattolin, P. Cogo, S. Palatron, F. Intini, S. Vedovato, G. Visintin, G. Verlato University of Padova, Padova, Italy

Background and aims: Osteopenia is an emerging problem in Neonatology. We studied if higher intakes of aminoacids (AA) provide bone growth advantages in preterm newborns without $A A$ intolerance.

Methods: Patients with birth weight $(\mathrm{BW})<1250$ $\mathrm{g}$ were randomly assigned from birth to receive lower (maximum 2,5 g/Kg/day, group L) or higher (maximum $4 \mathrm{~g} / \mathrm{Kg} /$ day, group $\mathrm{H}$ ) parenteral AA with the same non-proteic-energy and mineral intakes. By means of Quantitative Ultrasound (DBM-SonicBP Igea, Italy), to assess bone quality, we measured the second metacarpus Bone Trasmission Time (mcBTT; $\mu$ s) at birth, days 7, 14, 21, and 36 weeks of gestational age (GA) together with blood emogasanalysis, urea, calcium, phosphorus, creatinine, and urine output. Data are expressed as mean $\pm S D$; statistical significance analyzed by SPSS $13.0(p<0.05)$.

Results: We enrolled 47 vs 46 patients ( $L$ vs $H$ groups). No differences for basal clinical, bone, blood parameters and BW $(887,23 \pm 219.96$ vs $917,37 \pm 190,30 \mathrm{~g}$ ) were detected. Urea on day 7 was higher in group $\mathrm{H}(10,17 \pm 5,39$ vs $12,76 \pm 4,84 \mathrm{mmol} / /$; $p=0.04$ ); no differences were found between groups for bone (days $7,14,21$ ) and blood parameters at any other time. Nonetheless we found a positive correlation between mcBTT and blood phosphorus on day $21(r=0,42 ; p=0,001)$; higher $A A$ intakes in the first 3 weeks of life resulted in better mcBTT values at 36 weeks $\mathrm{GA}(0,40 \pm 0,04 \mu \mathrm{s}$ vs $0,43 \pm 0,05$ $\mu s ; p=0,04)$.

Conclusion: Early higher AA intakes could improve bone growth without short term AA intolerance. Further studies are required to evaluate long term bone growth. 